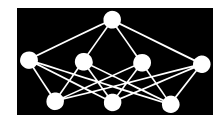

\title{
DYNAMICAL PROPERTIES OF FIRING PATTERNS IN THE HUBER-BRAUN COLD RECEPTOR MODEL IN RESPONSE TO EXTERNAL CURRENT STIMULI
}

\author{
J. Wang, S. Liu, H. Wang, Y. Zeng
}

\begin{abstract}
We have studied the role of external current stimuli in a four-dimensional Hodgkin-Huxley-type model of cold receptor in this paper. Firstly, we researched its firing patterns from direct current (DC) and alternating current (AC) stimuli. Under different values of DC stimulus intensity, interspike intervals (ISIs) with period-doubling bifurcation phenomena appeared. Second, research has shown that neurons are extremely sensitive to changes in the frequency and amplitude of the current used to stimulate them. As the stimulus frequency increased, discharge rhythms emerged ranging from burst firing to chaotic firing and spiking firing. Meanwhile, various phase-locking patterns have been studied in this paper, such as $p: 1(p>1), 1: q(q>1), 2: q(q>1)$ and $p: q(p, q>1)$, etc. Finally, based on the fast-slow dynamics analysis, codimension-two bifurcation analysis of the fast subsystem was performed in the parameter $\left(a_{s r}, B\right)$-plane. We mainly investigated cusp bifurcation, fold-Hopf bifurcation, Bogdanov-Takens bifurcation and generalized Hopf bifurcation. These results revealed the effect of external current stimuli on the neuronal discharge rhythm and were instructive for further understanding the dynamical properties and mechanisms of the Huber-Braun model.
\end{abstract}

Key words: firing patterns, phase-locking, interspike intervals (ISIs), firing rate, fast-slow dynamics analysis, Huber-Braun model

Received: June 26, 2014

DOI: $10.14311 / \mathrm{NNW} .2015 .25 .032$

Revised and accepted: May 30, 2015

\section{Introduction}

External current stimuli can change the dynamics of nonlinear systems qualitatively. And a lot of experiment data indicates that a neuron has a tendency to burst depending on many internal and external factors, such as all kinds of ion channels, ion concentration, depolarizing currents and membrane capacitance, etc.

\footnotetext{
* Jing Wang, Shenquan Liu - Corresponding author, School of Mathematics, South China University of Technology, Guangzhou 510640, China, E-mail: mashqliu@scut.edu.cn

${ }^{\dagger}$ Hongchu Wang, School of Mathematics Science, South China Normal University, Guangzhou 510631, China, E-mail: wang_hc@m.scnu.edu.cn

¥Yanjun Zeng, Biomedical Engineering Center, Beijing University of Technology, Beijing 100022, China, E-mail: yjzeng@bjut.edu.cn
} 
Neurons can produce electric signals which carry a mass of important adjusting and controlling information if they are stimulated [11]. Normally the dynamics of these systems are a little change when the parameters are slightly modified, but the situation will be a totally different at a critical point. The system would be transformed from a periodic pattern to chaotic pattern or from one periodic pattern to another periodic pattern $[15,16,18,21]$.

Here we study the Huber-Braun cold receptor model which was developed in 1998. Through use of a simplified Hodgkin-Huxley-type method, the model consists of two kinds of simplified sodium and potassium currents representing subthreshold oscillations and spike generation, respectively. The effect of Gaussian white noise and temperature had been deeply researched by Braun et al. According to the influence of temperature, there are two different types of transitions from tonic firing to bursting [2]. One appears in the high temperature region: when the temperature decreases, the dynamical properties of the system have no qualitative change. Another appears in the low temperature region: when the temperature increases, firing patterns transform from tonic firing to bursting. Their analyses demonstrate that temperature influences the slow, subthreshold currents in two different ways: when the temperature decreases, on the one hand, the wavelength increases, on the other hand, the subthreshold value of the oscillation amplitude decreases $[7,9]$. A special diversity between current and conductance noise have been discovered in particular situations $[6,8,14]$. However, in most cases, this difference is negligible.

The analogous data analysis reveals that adding external current stimuli can broaden the dynamical properties of the system to a large extent. Usually, the external current is added in the current balance equation to tune the model to different kinetic states. Previous numerical research on the firing patterns of HuberBraun cold receptor model under DC stimulus [17] showed that the model could display a rich variety of firing types, in addition, ISI sequences and the return map of ISIs are very diverse $[4,5,10,11,12,13,20]$. Furthermore, on this basis, we study the cosinusoidal modulation of the current injected into the model, which can also transform it from periodic firing to bursting.

In this paper, we focus on the properties of the firing patterns under DC and $\mathrm{AC}$ stimuli. Besides we also analyze the neuronal average firing rate, phase-locking rhythms, the ISIs bifurcation diagrams, and the return map of ISIs. With the support of numerical simulation and nonlinear dynamical theory, many firing patterns are observed and analyzed. Moreover, under external cosinusoidal stimulus, a lot of phase-locking patterns emerge. At last, codimension-two bifurcation analysis is utilized to better understand the combined influences of the slow repolarizing variable $a_{s r}$ and the DC stimulus intensity $B$ on the dynamical behavior of the Huber-Braun model.

The paper is constituted as follows. In Section 2, we describe a simplified Hodgkin-Huxley type model of a cold receptor and the corresponding numerical simulation method. In Section 3, the dynamical behavior of this model stimulated by external electrical stimuli is investigated. Section 4 shows the codimensiontwo bifurcation analysis of the fast subsystem in the control parameter $\left(a_{s r}, B\right)$ plane based on the fast-slow dynamics analysis. Finally, conclusions are given in Section 5. 


\section{Model and method}

We apply a simplified Hodgkin-Huxley-type method as previously depicted $[1,2,3]$. In short, this model is composed of two minimal sets of depolarizing and repolarizing currents which are activated at different voltage levels and with different delays. It is a single compartment model with the following membrane equation:

$$
C_{\mathrm{M}} \frac{\mathrm{d} V}{\mathrm{~d} t}=-g_{l}\left(V-V_{l}\right)-I_{d}-I_{r}-I_{s d}-I_{s r}-I_{\mathrm{ext}},
$$

where $V$ is the membrane voltage and $C_{\mathrm{M}}$ is the membrane capacitance. The leakage current is given by $g_{l}\left(V-V_{l}\right) ; I_{d}$ represents the fast depolarizing spikecurrent $I_{N a} ; I_{r}$ represents the fast repolarizing spike-current $I_{K} ; I_{s d}$ stands for the slow depolarizing current $I_{N a P} ; I_{s r}$ stands for the slow repolarizing current $I_{K(\mathrm{Ca})}$; $I_{\text {ext }}$ is the external current.

The voltage-dependent currents are computed using the following equations $(i=d, r, s d, s r)$ :

$$
\begin{aligned}
I_{i} & =\rho g_{i} a_{i}\left(V-V_{i}\right), i=d, r, s d, s r, \\
a_{i \infty} & =\frac{1}{1+\exp \left(-s_{i}\left(V-V_{0 i}\right)\right)}, i=d, r, s d, \\
\frac{d a_{r}}{d t} & =\frac{\phi\left(a_{r \infty}-a_{r}\right)}{\tau_{r}}, \\
\frac{d a_{s d}}{d t} & =\frac{\phi\left(a_{s d \infty}-a_{s d}\right)}{\tau_{s d}}
\end{aligned}
$$

where $V_{i}$ is the equilibrium potential, $g_{i}$ is the maximal conductances. The quantities $V_{0 i}$ and $s_{i}$ are half-activation potentials and slopes of the steady-state activation curves, respectively. $\tau_{i}$ is the voltage independent activation time-constant.

Instantaneous activation of the fast depolarizing current is represented by

$$
a_{d}=a_{d \infty}
$$

The slow repolarizing current $a_{s r}$ is coupled directly to the slow depolarizing current $I_{s d}$ by means of

$$
\frac{d a_{s r}}{d t}=\frac{\phi\left(-\eta I_{s d}-k a_{s r}\right)}{\tau_{s r}}
$$

where $\eta$ is a coupling constant and $k$ is a relaxation factor.

Temperature dependencies are described by the scaling parameters and for the maximum conductances and the time constants, respectively ( $T$ : temperature, $T_{0}$ : reference temperature):

$$
\rho=1.3^{\frac{T-T_{0}}{10}}, \quad \phi=3.0^{\frac{T-T_{0}}{10}} .
$$

The external current stimulus is implemented in the following form:

$$
I_{\text {ext }}=B+A \cos (2 \pi f t),
$$

when $A=0$, the external current stimulus $I_{\text {ext }}$ is a DC form, while $B=0$ makes the $I_{\text {ext }}$ a AC form. 


\begin{tabular}{rccccc}
\hline Parameter & Value & Parameter & Value & Parameter & Value \\
\hline$V_{l}[m V]$ & -60 & $g_{l}[n s]$ & 0.1 & $C_{\mathrm{M}}[\mu F]$ & 1.0 \\
$V_{d}[m V]$ & 50 & $g_{d}[n s]$ & 0.91 & $V_{0 d}[m V]$ & -25 \\
$V_{r}[m V]$ & -90 & $g_{r}[n s]$ & 1.21 & $V_{0 r}[m V]$ & -25 \\
$V_{s d}[m V]$ & 50 & $g_{s d}[n s]$ & 0.15 & $V_{0 s d}[m V]$ & -40 \\
$V_{s r}[m V]$ & -90 & $g_{s r}[n s]$ & 0.24 & $T_{0}\left[{ }^{\circ} C\right]$ & 25 \\
$\tau_{s d}[m s]$ & 80 & $\tau_{s r}[m s]$ & 160 & $\tau_{r}[m s]$ & 16 \\
$s_{r}$ & 0.25 & $s_{s d}$ & 0.09 & $s_{d}$ & 0.25 \\
$\eta$ & 0.012 & $k$ & 0.17 & & \\
\hline
\end{tabular}

Tab. I Parameter values for the Huber-Braun cold receptor model.

The average firing rate of the neuron is defined as

$$
f_{R}=\frac{N_{s}}{T_{\text {integ }}},
$$

where $N_{s}$ is the number of spikes and $T_{\text {integ }}$ is the integration time which the neuron fires during. If the neuron is stimulated by periodic input with a frequency $f$, we adopt $T_{\text {integ }}$ as an integer product of the time period $1 / f$.

Some parameters are given in Tab. I. The calculation of this model was programed in Python by using a fourth-order Runge-Kutta algorithm. One-parameter bifurcation diagrams were performed using XPPAUT software, and codimensiontwo bifurcation diagram was computed using MATCONT software. Other diagrams were drawn using an open source library in Python named Matplotlib. In our simulation, we set the initial value of membrane potential as $-60[\mathrm{mV}]$, while initial values of other three variables were set as the values of the steady-state activation. To avoid the influence of initial values, we deliberately discarded the values $0-20[\mathrm{~s}]$, and analyzed the action potential values during $20-40[\mathrm{~s}]$. In this paper, we didn't focus on the effect of temperature on the model, so we made the temperature remain stable; that is, $T=T_{0}$. The values of membrane potential were calculated with a time step of 0.1 [ms]; bifurcation diagrams with a time integration of $0.05[\mathrm{~ms}]$. Simulated results have been repeatedly verified.

\section{Simulation results}

\subsection{Variation of firing patterns under DC stimulus}

In neurons, DC stimulus could be considered as a constant stimulus or a compound synaptic current. In this part, we study the role of DC stimulus intensity on neuronal firing patterns. Studies have shown that phase diagrams can be used to describe the periodic number of the corresponding firing patterns [20]. We draw the phase diagram of the slow depolarizing variable $a_{s d}$ with respect to the neuronal action potentials. As the value of DC stimulus increases, we get the typical discharge sequences as shown in Fig. 1. In Fig. A we can see, the firing patterns present a period-1 pattern when $B=0$, as shown in [17]. As $B$ increases to 0.12 , the 
Wang J. et al.: Dynamical properties of firing patterns...
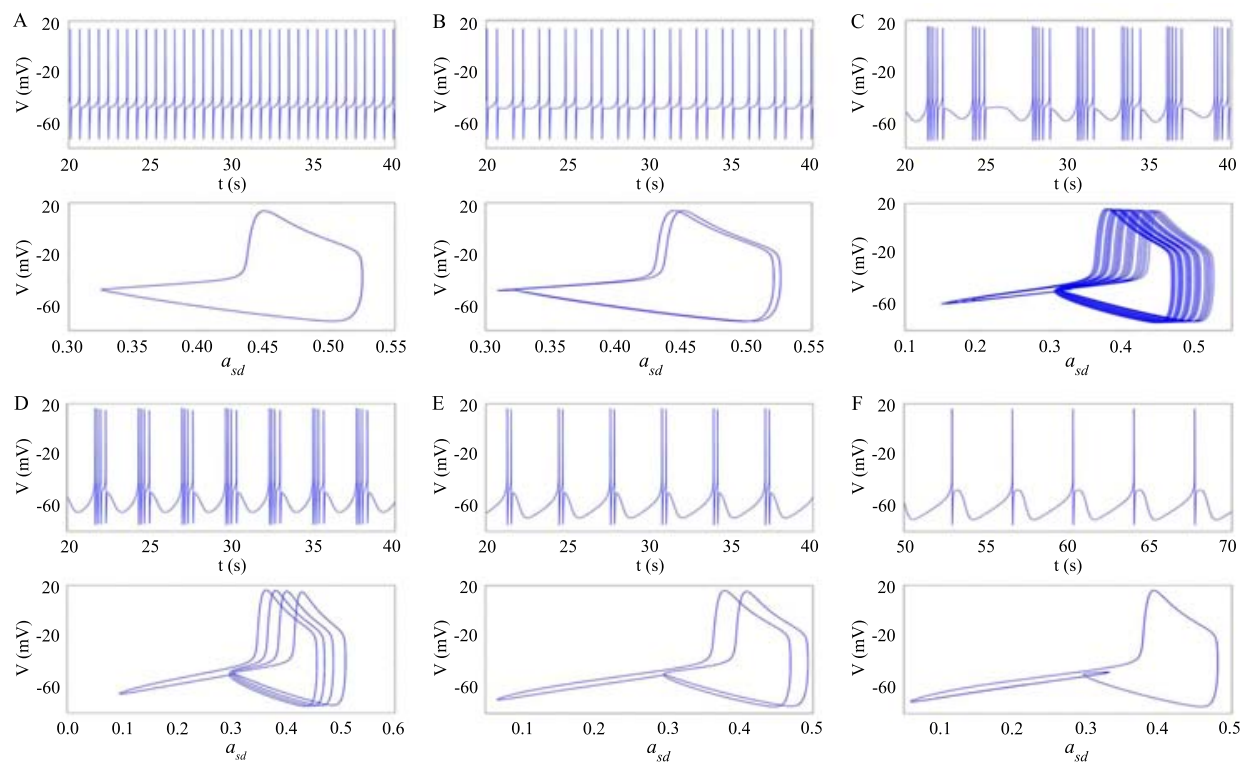

Fig. 1 Neuronal action potentials and the corresponding firing periods under different values of $B$. From $A-F$, the value of $B$ is $0,0.12,0.4,0.8,1.2$, and 1.25 [nA], respectively. In each diagram, the upper part represents the neuronal action potentials, and the lower part indicates the corresponding firing number of the model. Different values of $B$ can induce different firing patterns and firing numbers.

neuron fires a period- 2 pattern, while a period- 4 pattern emerges when $B$ increases to 0.1293 . Due to this change in periodic number from one period to two and then from two to four periods, it is apparent that this is a period-doubling bifurcation phenomenon, which can also be observed in the ISIs bifurcation diagram of Fig. 2a. After the period-doubling cascade, the neuron model enters into a chaotic firing pattern and multi-period firing pattern alternately. After that, a period-4 firing pattern appears when $B=0.8$, when $B$ continues to increase to 1.0 , there is a period-3 pattern, and the neuron model displays a period- 2 when $B=1.2$. Finally, the neuron model reaches a resting state after first producing a period-1 firing pattern.

The particular transition modes between these firing patterns with the linear increase of DC stimulus intensity is illustrated in Fig. 2a, which can obviously show how these firing patterns transform from one to another. We also give the variation in neuronal average firing rate as a function of DC stimulus intensity in Fig. 2b, which indicates that for a larger DC stimulus intensity, the average firing rate is somewhat lower. That is to say, the average firing rate of the neuron model can be modulated by external DC stimulus. Furthermore, the average firing rate changes irregularly in chaotic areas and decreases suddenly at the points when the number of period changes. 

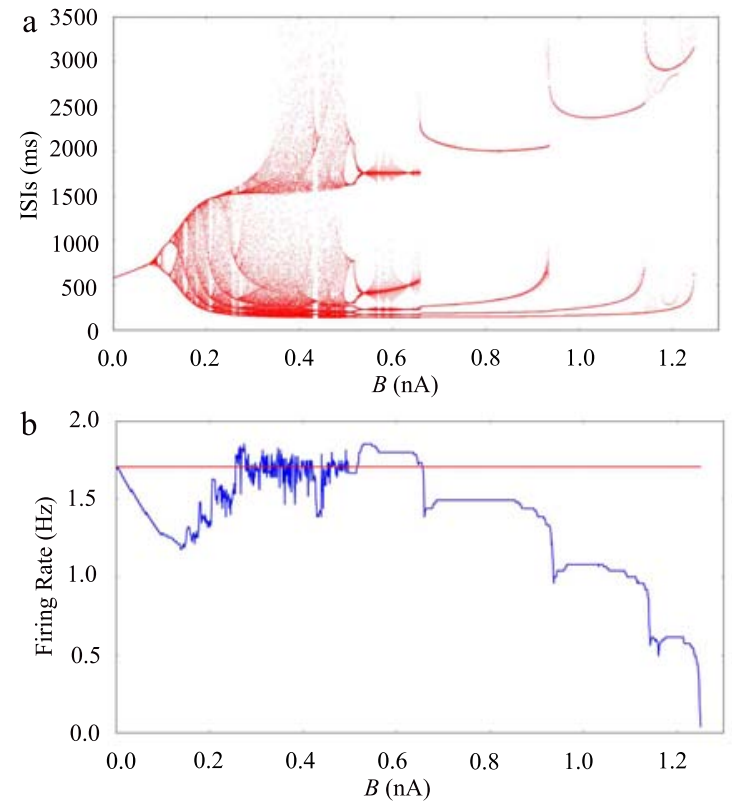

Fig. 2 Interspike intervals (ISIs) and the average firing rate as $B$ changes. a: The ISIs bifurcation diagram; b: Firing Rate. The red horizontal line in diagram $b$ represents the values of firing rate without external current stimuli.

\subsection{Variation of firing patterns under AC stimulus}

\subsubsection{Response of the neuron to cosinusoidal AC stimulus}

As we know, AC stimulus could be treated as a periodic stimulus input or may result from the synchronized activity in connected brain areas. In this section, we choose the form of cosinusoidal AC stimulus to research firing patterns and the firing rate when varying the stimulus frequency. Fig. 3 is the diagram of ISIs with respect to frequency for stimulus current at $A=0.4$ and $A=1.0$. It is obvious that amplitudes of 0.4 and 1.0 can inspire the model to produce discharge phenomenon at a large range of frequencies. By increasing the stimulus frequency, ISIs sharply shorten from thousands of milliseconds to hundreds of milliseconds and the model discharges from bursting to tonic spiking and finally reaches a steady state. Furthermore, the firing patterns alternate between periodic discharge and chaotic firing. We may draw a conclusion from the above analysis that the neuron is particularly sensitive to change in the stimulus frequency.

Fig. 4 is the diagram of the average firing rate with respect to frequency for stimulus current at $A=0.4$ and $A=1.0$. The average firing rate does not monotonically change as stimulus frequency changes, but achieves a maximum value (when $A=0.4, f=0.24, f_{R}=4.229$ or when $\left.A=1.0, f \in[6.32,6.36], f_{R}=6.343\right)$. The neuron fires at a high rate and discharges more in a stimulus cycle. Subsequently, the average firing rate first increases and then decreases periodically as the frequency increases. And the secondary maximum still exists, even at a relatively large amplitude. 
Wang J. et al.: Dynamical properties of firing patterns...
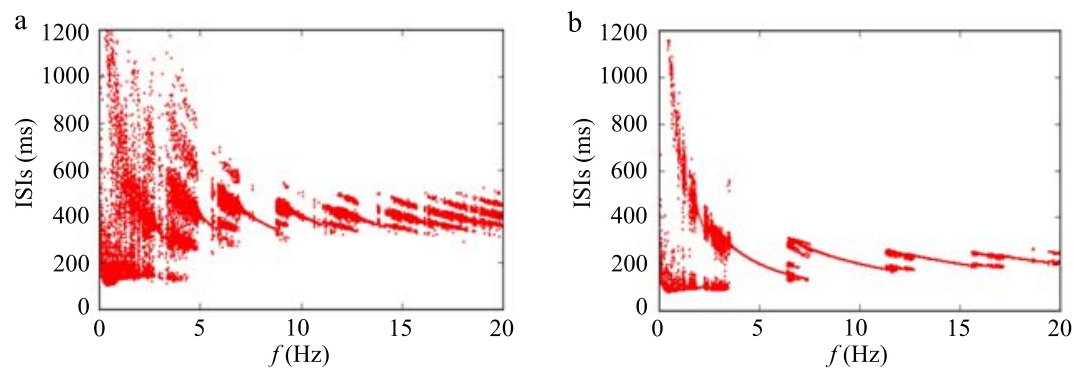

Fig. 3 Interspike intervals (ISIs) with respect to stimulus frequency $f$. $a$ : $A=0.4$; $b: A=1.0$. With the increase of the stimulus frequency, ISIs reduce quickly.
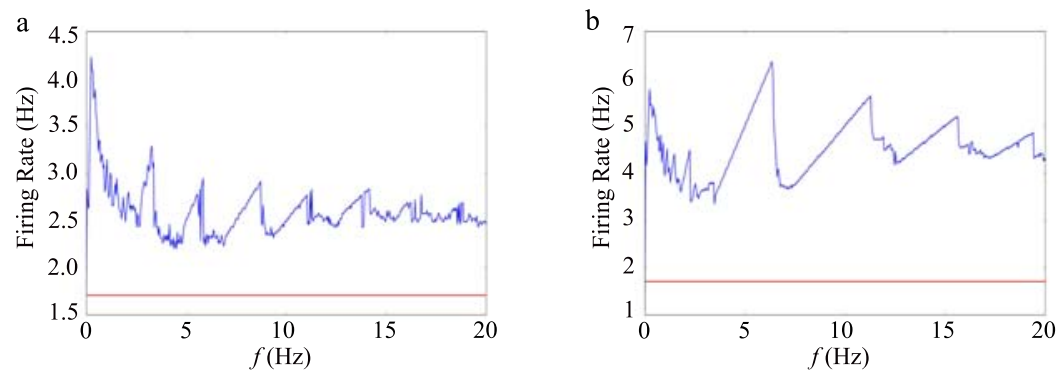

Fig. 4 Firing rate with respect to stimulus frequency $f . a: A=0.4 ; b: A=1.0$. The red horizontal line represents the values of firing rate without external current stimuli.

We choose $I_{\text {ext }}=A \cos (2 \pi f t)$ where $A=0.4$. First of all, we propose the definition of $p: q$ phase-locking: a periodic oscillation is defined as $p$ action potentials produced by $q$ cycle motivations [19]. Here, we use an abbreviation RPL (ratio of phase-locking) to denote it. With a stimulus frequency range $f \in[0.1,20][\mathrm{Hz}]$ increasing in steps of $0.1[\mathrm{~Hz}]$, the basic information of the phase-locking ratios for the Huber-Braun model is illustrated in Tab. II.

In Tab. II, with the frequency ranges $f \in[0.1,2.9][\mathrm{Hz}], f \in[3,3.3][\mathrm{Hz}]$, and $f \in[3.4,6.9] \cup[7.4,20][\mathrm{Hz}]$, three types of phase-locking ratios appear, i.e. alternating between chaos and $p: 1(p>1), 1: 1$, from $1: q$ to $2: q(q>1)$ passing through the chaotic region, respectively. In the $[7,7.3]$ region, the phase-locking ratio appears 6:18 mode. Rather than showing all of the frequencies, listed these ranges were picked to show the entrainment modes, ISIs and the return maps of ISIs. Here the frequencies $0.8[\mathrm{~Hz}], 7.2[\mathrm{~Hz}], 10[\mathrm{~Hz}]$ and $11.4[\mathrm{~Hz}]$ are selected to show the characteristics of RPL sections $p: 1,6: 18$, chaos, and $2: q$, respectively. From Fig. 5, we find that ISIs are periodic in the phase-locking patterns whose return maps are regular too, while ISIs are out-of-order, when the corresponding return map is irregular too. Besides the frequencies mentioned above, simulations were taken in all the other frequencies, and we found: in $p: 1$ section, as the frequency increases, the value of $p: 1$ decreases, and the number of maximal ISIs 


\begin{tabular}{rcccccc}
\hline$f$ & 0.1 & 0.2 & 0.3 & $0.4-0.7$ & 0.8 & $0.9-1.4$ \\
RPL & chaos & $19: 1$ & $14: 1$ & chaos & $4: 1$ & chaos \\
\hline$f$ & 1.5 & $1.6-2.9$ & $3-3.3$ & $3.4-5.1$ & $5.2-5.8$ & $5.9-6.9$ \\
RPL & $2: 1$ & chaos & $1: 1$ & chaos & $1: 2$ & chaos \\
\hline$f$ & $7-7.3$ & $7.4-8.7$ & $8.8-10.1$ & $10.2-11.2$ & $11.3-11.6$ & $11.7-13.5$ \\
RPL & $6: 18$ & $1: 3$ & chaos & $1: 4$ & $2: 9$ & chaos \\
\hline$f$ & $13.6-14.1$ & 14.2 & $14.3-14.5$ & $14.6-16.5$ & 16.6 & $16.7-18.7$ \\
RPL & $1: 5$ & chaos & $2: 11$ & chaos & $1: 5$ & chaos \\
\hline$f$ & 18.8 & $18.9-19.1$ & 19.2 & $19.3-20$ & & \\
RPL & $1: 6$ & chaos & $2: 13$ & chaos & & \\
\hline
\end{tabular}

Tab. II The $p$ : q phase-locking of the Huber-Braun model with the stimulus frequency.

is also decreasing, which holds true for $2: q$ section; in $1: q$ section, the value of $1: q$ decreases when the frequency increases; ISIs are periodic in phase-locking patterns and chaotic in chaotic patterns. So we can come to the conclusion that the frequency of the external current stimulus plays a significant role in transitions, in the Huber-Braun model, from periodic patterns to chaotic patterns, or from one periodic pattern to another periodic pattern.

\subsubsection{Response of the neuron to cosinusoidal AC stimulus of amplitudes dependent on frequency}

AC stimulus of amplitudes dependent on frequency may be understood as some kind of current noise. For the Huber-Braun model, many literatures related to the Gaussian white noise have been extensively investigated $[1,3,4,5,6,8,14]$. Here we propose another current noise which is easier to operate in the experiment. Compared with the Gaussian white noise, the current noise implemented here could induce more abundant dynamical phenomena.

The diagrams in Fig. 6 illustrate ISIs bifurcation phenomena that plot ISIs versus frequency for the stimulus current $I_{\text {ext }}=(A /(2 \pi f)) \cos (2 \pi f t)$ at $A=0.4$ and $A=1.0$. It is totally different from the normal type of $\mathrm{AC}$ stimulus as previously described. Bifurcation phenomena are more varied. A special transition pattern (from multi-period bursting to chaos to regular spiking) periodically appears if frequency is increased. This periodic variation phenomenon may imply some potential roles in sensory information encoding and it deserves further experimental investigation into oscillatory activity with the addition of noise.

The diagrams in Fig. 7 illustrate the firing rate versus stimulus frequency for the stimulus current $I_{\text {ext }}=(A /(2 \pi f)) \cos (2 \pi f t)$ at $A=0.4$ and $A=1.0$. Compared with Fig. 4 , the changes are relatively simple. We achieve a maximum value (when $A=0.4, f_{R}=9.829$ or when $\left.A=1.0, f_{R}=13.629\right)$ when $f=0.016$. Despite the different stimulus amplitude, the maximum is obtained at the same point. After the extreme value point, the average firing rate decreases when the frequency is increasing, without changing periodically. What's more, in this circumstance, the 
Wang J. et al.: Dynamical properties of firing patterns...
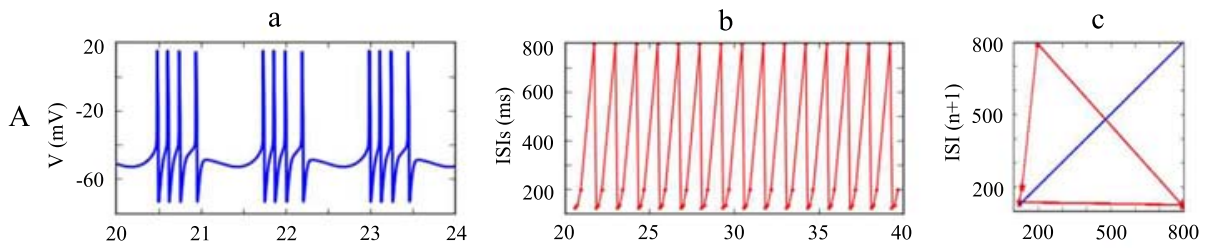

$\mathrm{B}$
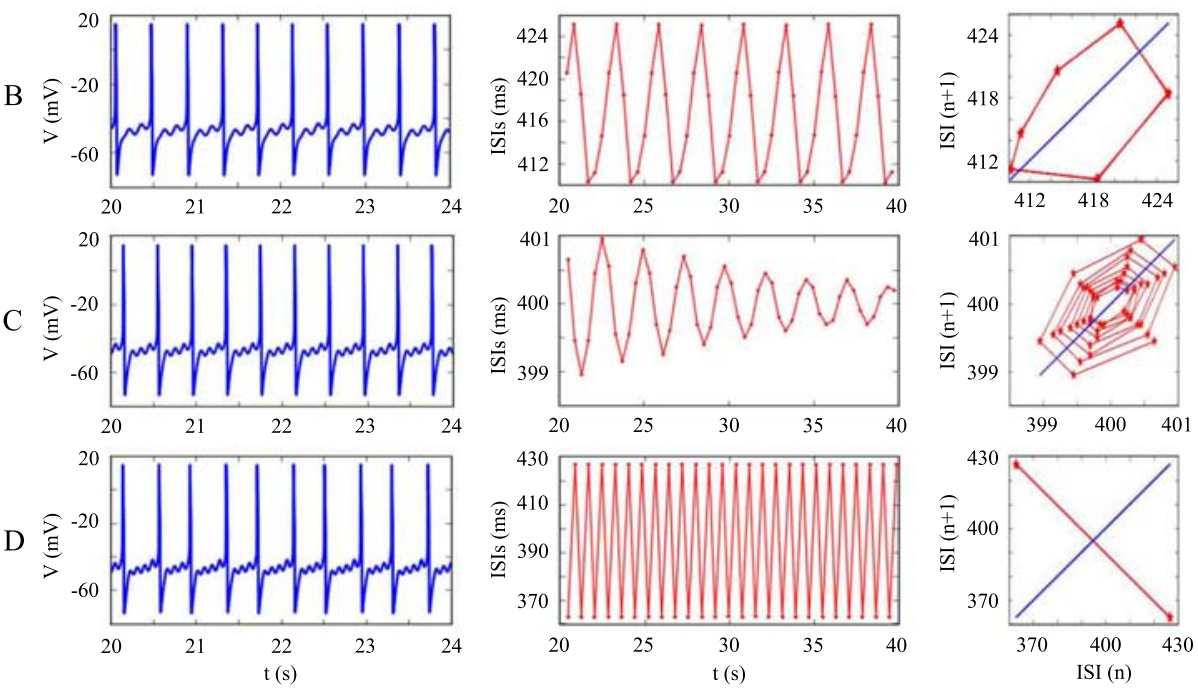

Fig. 5 Fixing amplitude at $A=0.4$, we get the typical discharge and ISIs sequences. (a) plots membrane potentials during a 4 second steady-state period. (b) shows the evolution of ISIs. The return map of ISIs is given in (c). A: Entrainment mode of $p: 1$ at stimulus frequency of $0.8[\mathrm{~Hz}] ;$ B: Entrainment mode of $6: 18$ at stimulus frequency of 7.2 [Hz]; C: Entrainment mode of chaos at stimulus frequency of 10 [Hz]. D: Entrainment mode of $2: q$ at stimulus frequency of $11.4[\mathrm{~Hz}]$. The $45^{\circ}$ line of the third column indicates the positions of identical values which reflects regular tonic firing.
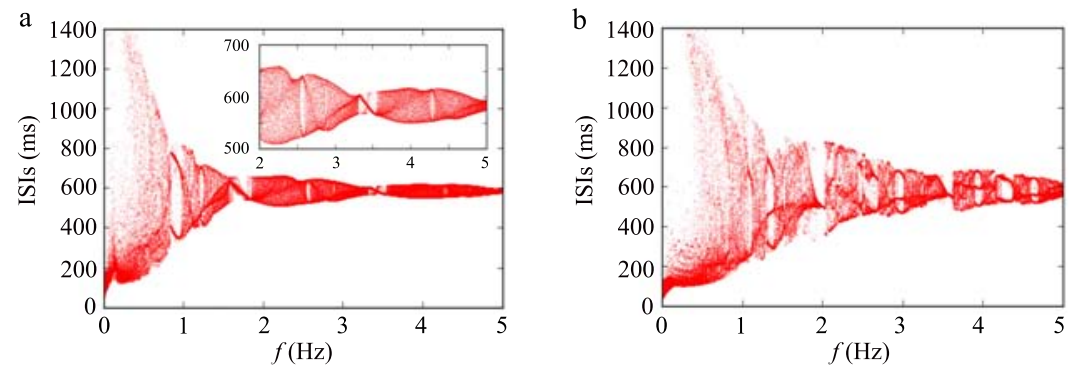

Fig. 6 Interspike intervals (ISIs) with respect to stimulus frequency $f$. a: $A=0.4$; $b: A=1.0$. The diagram in the top right corner is an enlargement of diagram $a$. With the increase of the stimulus frequency, ISIs reduce quickly. 

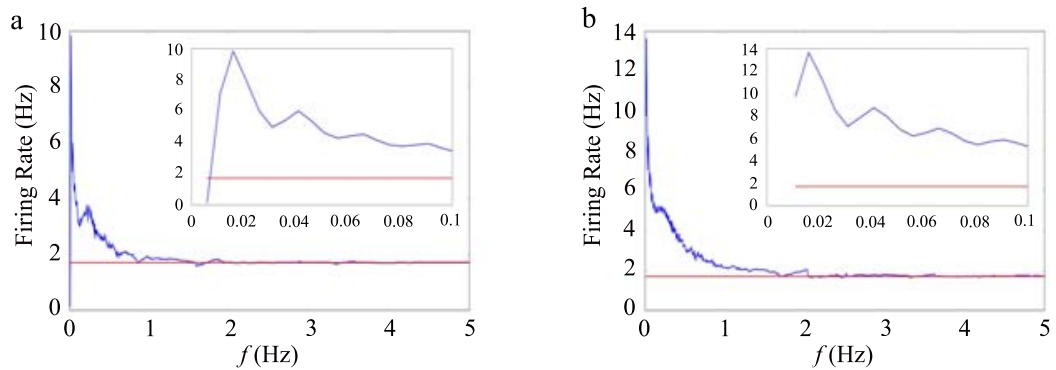

Fig. 7 Firing rate with respect to stimulus frequency $f . a: A=0.4 ; b: A=1.0$. The diagrams in the top right corner is an enlargement of diagram a and $b$, respectively. With the increase of the stimulus frequency, the firing rate of the neuron increases first, then reduces to 1.714 which is quite close to the firing rate without external current stimuli, and finally reaches a steady state. The red horizontal line represents the values of firing rate without external current stimuli.

neuron is more prone to discharge in an ultra-low frequency range (0 to $1.0[\mathrm{~Hz}])$. The value of the initial discharge is also different. When $A=0.4$, the minimal firing rate is $0.143[\mathrm{~Hz}]$, which is very close to zero. Nevertheless, when $A=1.0$, the situation is rather different: the minimal firing rate jumps to 9.771 [Hz]. This indicates that the changes in amplitude influence the initial value of the discharge to a great degree.

\section{Fast-slow dynamics analysis}

In this section, we use the fast-slow dynamic analysis to investigate the dynamical properties of the Huber-Braun model. The variable of the slow repolarizing variable $a_{s r}$ is relatively slow, with the time constant $\tau_{s r}=160$ [ms]. Therefore the fast-slow method can be implemented in the condition that $a_{s r}$ is choosen as a slowly-varying bifurcation parameter.

Codimension-two bifurcation analysis of the fast subsystem Eqs. (1), (4) and (5) is performed in the $\left(a_{s r}, B\right)$ plane, as shown in Fig. 8. There are four important codimension-two bifurcation points, namely, the cusp (CP), fold-Hopf ( $\mathrm{ZH})$, Bogdanov-Takes (BT) and generalized Hopf (GH) bifurcations. Some data related to these four special points are listed in Tab. III. The bifurcation curves in Fig. 8 display how the one-parameter bifurcations (including the supercritical Hopf (suph), subcritical Hopf (subh), fold $\left(f_{1}\right.$ and $\left.f_{2}\right)$ bifurcations of equilibrium point, and the fold limit cycle $(l)$ bifurcations of limit cycles) vary with the parameters.

Each point in the fold bifurcation curves $f_{1}$ and $f_{2}$ is an equilibrium point with a zero eigenvalues $\lambda_{1}=0$ and the other two eigenvalues have nonzero real parts. In general, the restriction of the fast subsystem to the center manifold has the normal form

$$
\xi^{\prime}=a \xi^{2}+O\left(|\xi|^{3}\right), \xi \in \mathbb{R}^{1} .
$$

When the parameters $a_{s r}$ and $B$ vary, two bifurcation curves $f_{1}$ and $f_{2}$ coalesce and disappear at the cusp point CP $(0.8364,-4.7953)$ where the eigenvalues are $\lambda_{1}=0$, 
Wang J. et al.: Dynamical properties of firing patterns...

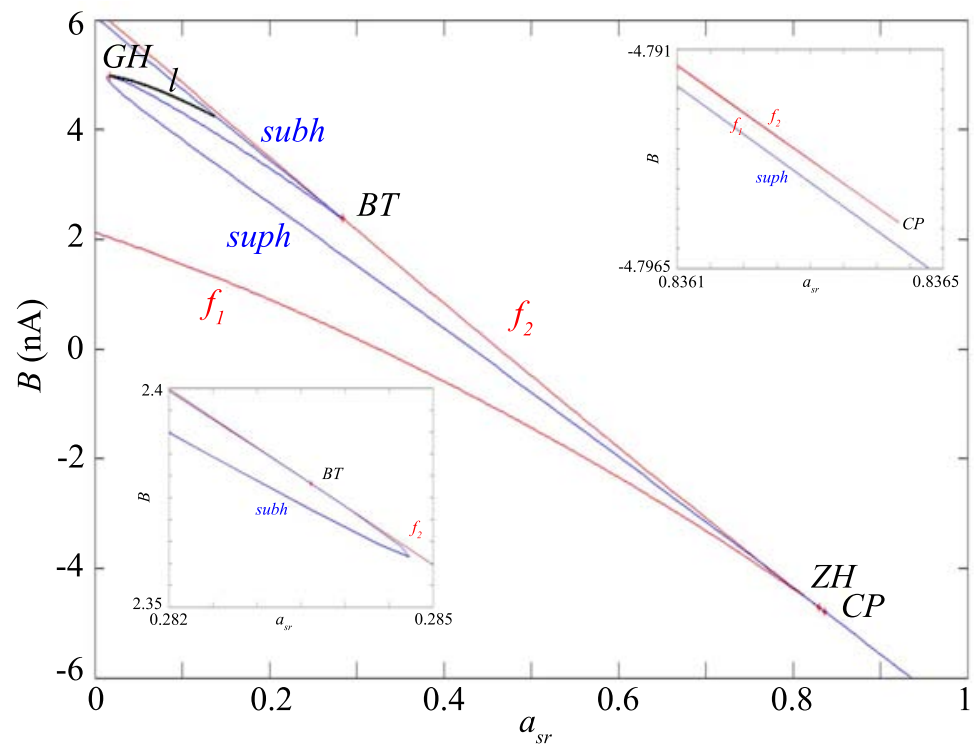

Fig. 8 The codimension-two bifurcation analysis of the fast subsystem with respect to the parameters $a_{s r}$ and $B$. The labels indicate the following bifurcations: $C P=$ cusp, $Z H=$ fold-Hopf, BT =Bogdanov-Takens, $G H=$ generalized Hopf $($ or Bautin). Besides, suph and subh separately represent the supercritical and subcritical Hopf bifurcation curves; $f_{1}$ and $f_{2}$ are the fold bifurcation curves; l corresponds to the fold bifurcation of limit cycles. The diagram in the top right corner is an enlargement near CP; the diagram in the bottom left corner is an enlargement near BT.

\begin{tabular}{rcc}
\hline Points & Parameter values $\left(a_{s r}, B\right)$ & Eigenvalues $\lambda_{1}, \lambda_{2}, \lambda_{3}$ \\
\hline CP & $(0.8364,-4.7953)$ & $\lambda_{1}=0, \lambda_{2,3}=-0.0472998 \pm 0.118635 i$ \\
ZH & $(0.8295,-4.7121)$ & $\lambda_{1}=0, \lambda_{2,3}= \pm 0.1223 i$ \\
BT & $(0.2836,2.3782)$ & $\lambda_{1,2}=0, \lambda_{3}=1.05089$ \\
GH & $(0.0169,4.9733)$ & $\lambda_{1}=0.128002, \lambda_{2,3}= \pm 0.0346361 i$ \\
\hline
\end{tabular}

Tab. III Data related to the special points.

$\lambda_{2,3}=-0.0472998 \pm 0.118635 \mathrm{i}$. At this point, the coefficient $a$ of normal form in Eq. (11) vanishes; namely, $a=0$. Then, the restriction of the fast subsystem to the center manifold becomes the normal form

$$
\xi^{\prime}=c \xi^{3}+O\left(|\xi|^{4}\right), \xi \in \mathbb{R}^{1}
$$

where $c=-2.026808 \times 10^{-5}$. 
Near the point CP, the Eqs. (1), (4) and (5) are locally topologically equivalent to the normal form

$$
\left\{\begin{array}{c}
\xi^{\prime}=\beta_{1}+\beta_{2} \xi+\sigma \xi^{3}, \\
\eta_{-}^{\prime}=-\eta_{-}, \\
\eta_{+}^{\prime}=\eta_{+},
\end{array}\right.
$$

where $\sigma=\operatorname{sign}(c)=-1, \eta_{ \pm} \in \mathbb{R}^{1}, \beta_{1,2} \in \mathbb{R}$.
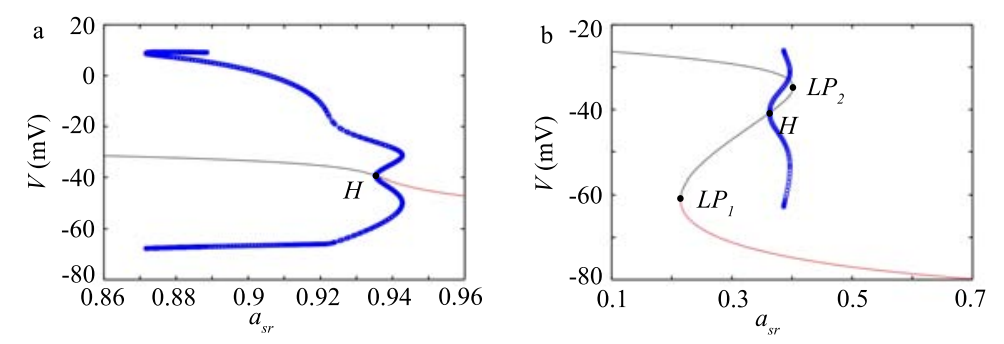

Fig. 9 Typical one-parameter bifurcation diagrams of the fast subsystem with the variation of $B$. a: $B=-6[n A] ; b: B=0.8[n A]$. Stable steady states are marked by red curves; unstable steady states by black curves. The blue cycles indicate the limit cycles. $H$ stands for the supercritical Hopf bifurcation. $L P_{1}$ and $L P_{2}$ represent the fold bifurcations.

With further varying of the parameters, a fold-Hopf (ZH) bifurcation emerges at the point labeled ZH $(0.8295,-4.7121)$ with one zero eigenvalue $\lambda_{1}=0$ and a pair of complex conjugate eigenvalues $\lambda_{2,3}= \pm 0.1223 i$. Near the point ZH, the Eqs. (1), (4) and (5) are locally topologically equivalent to the normal form

$$
\left\{\begin{array}{c}
\xi^{\prime}=\beta_{1}+\xi^{2}+s \rho^{2}, \\
\rho^{\prime}=\rho\left(\beta_{2}+\theta \xi\right),
\end{array}\right.
$$

where $s=-1, \theta=-280.6281$. The point $\mathrm{ZH}$ is the intersection point of the supercritical Hopf bifurcation curve suph and the upper fold bifurcation curve $f_{2}$.

A Bogdanov-Takes bifurcation takes place at the point labeled BT $(0.2836$, 2.3782) with two zero eigenvalues $\lambda_{1,2}=0$ and one real eigenvalues $\lambda_{3}=1.05089$. Near the point BT, the Eqs. (1), (4) and (5) are locally topologically equivalent to the normal form

$$
\left\{\begin{array}{c}
\eta_{1}^{\prime}=\eta_{2}, \\
\eta_{2}^{\prime}=\beta_{1}+\beta_{2} \eta_{1}+\eta_{1}^{2}+s \eta_{1} \eta_{2},
\end{array}\right.
$$

where $a=2.275099 \times 10^{-5}, b=1.790721 \times 10^{-4}, s=\operatorname{sign}(a b)=1$. The point BT is the tangency point of subcritical Hopf bifurcation curve subh and the upper fold bifurcation curve $f_{2}$.

A generalized Hopf bifurcation takes place at the point labeled GH (0.0169, 4.9733) with one real eigenvalue $\lambda_{1}=0.128002$ and a pair of complex conjugate eigenvalues $\lambda_{2,3}= \pm 0.0346361 i$. At the point $\mathrm{GH}$, the first Lyapunov coefficient vanishes; that is, $l_{1}=0$. Near the point GH, the Eqs. (1), (4) and (5) are locally topologically equivalent to the normal form

$$
\left\{\begin{array}{c}
z^{\prime}=\left(\beta_{1}+i\right) z+\beta_{2} z|z|^{2}+s z|z|^{4}, z \in \mathbb{C}^{1} \\
\eta_{-}^{\prime}=-\eta_{-},
\end{array}\right.
$$


where $l_{2}$ is the second Lyapunov coefficient and $s=\operatorname{sign}\left(l_{2}\right)=\operatorname{sign}(-2.119903 \times$ $\left.10^{-3}\right)=-1$. The point GH separates the branch of the supercritical Hopf (suph) and subcritical Hopf (subh) bifurcations.

When the parameter $B$ increases from the down to upper in Fig. 8, the oneparameter bifurcation diagram changes from a monotone curve (Fig. 9a) to a $Z$ shaped curve (Fig. 9b). Furthermore, the type of equilibrium points is also changing.

\section{Conclusions}

Based on the Huber-Braun model, the impacts of external current stimuli (DC and $\mathrm{AC}$ ) on neuronal firing patterns are researched. In the present research, our main findings are:

(1) The model exhibits abundant firing patterns under DC stimulus. With the increase of DC stimulus intensity $B$, a period-doubling bifurcation phenomenon takes place. After the period-doubling cascade, the model enters into chaos. When $B \geq 0.66[\mathrm{nA}]$, the model exhibits an inverse period-adding bifurcation. What's more, the firing rate changes irregularly in chaotic areas and decreases suddenly at the point when the discharge pattern transforms from one to another.

(2) For cosinusoidal AC stimulus, at first, interspike intervals (ISIs) sharply shorten from thousands of milliseconds to hundreds of milliseconds by increasing the stimulus frequency. Furthermore, periodic firing and chaotic firing alternately appear; the firing rate first increases and then decreases periodically. At last, a lot of phase-locking patterns have been observed. Similar phenomena are also found by Wang et al. [19] in Hodgkin-Huxley model, but no one puts forward these phenomena in the Huber-Braun model so far.

(3) We propose another current noise in a manner of external current stimulus. With the addition of noise, a special transition mode (from multi-period bursting to chaos to regular spiking) periodically appears. Besides, the maximum value of the firing rate is independent on the stimulus amplitude.

(4) By using codimension-two bifurcation analysis of the fast subsystem, we give a theoretical description of the basic mechanism. We not only detect the properties of the codimension-two bifurcation points, but also provide the normal form of the fast subsystem to the center manifold. Research on this model rarely involved in this aspect. Our work intends to fill this gap.

The results in this paper can be used to guide a further investigation of dynamical properties of the model in response to external current stimuli, allowing one to predict the dynamics emerging in distinct types of input information. Our investigation may have significant implications for further study of the effect of external current stimuli on an organism and may establish a possible instruction on further biological experiments.

\section{Acknowledgement}

This work was supported by the National Natural Science Foundation of China under Grant No. 11172103. 


\section{References}

[1] BRAUN H.A., HUBER M.T., DEWALD M., SCHÄFER K., VOIGT K. Computer simulations of neuronal signal transduction: the role of nonlinear dynamics and noise. International Journal of Bifurcation and Chaos. 1998, 8(5), pp. 881-889, doi: 10.1142/S0218127498000681.

[2] BRAun H.A., Huber M.T., ANTHes N., VOigT K., NEIMAN A., PEI X., MOSS F. Interactions between slow and fast conductances in the Huber/Braun model of cold-receptor discharges. Neurocomputing. 2000, 32, pp. 51-59, doi: 10.1016/S0925-2312(00)00143-0.

[3] BRAUn H.A., HUber M.T., ANTheS N., VOIGT K., NEIMAN A., PEI X., MOSS F. Noise-induced impulse pattern modifications at different dynamical period-one situations in a computer model of temperature encoding. Biosystems. 2001, 62(1), pp. 99-112, doi: 10 . 1016/S0303-2647 (01) 00140-X.

[4] BRAUN H.A., VOIGT K., HUBER M.T. Oscillations, resonances and noise: basis of flexible neuronal pattern generation. Biosystems. 2003, 71(1), pp. 39-50, doi: 10.1016/ S0303-2647 (03) 00108-4.

[5] DU Y., LU Q., WANG R. Using interspike intervals to quantify noise effects on spike trains in temperature encoding neurons. Cognitive neurodynamics. 2010, 4(3), pp. 199-206, doi: 10. 1007/s11571-010-9112-2.

[6] Finke C., VOLLMER J., POSTNOVA S., BRAUN H.A. Propagation effects of current and conductance noise in a model neuron with subthreshold oscillations. Mathematical biosciences. 2008, 214(1), pp. 109-121, doi: 10.1016/j.mbs.2008.03.007.

[7] FINKE C., FREUND J.A., ROSA E., BRAUN H.A., FEUDEL U. On the role of subthreshold currents in the Huber-Braun cold receptor model. Chaos: An Interdisciplinary Journal of Nonlinear Science. 2010, 20(4), 045107, doi: 10.1063/1.3527989.

[8] Finke C., POSTNOVA S., ROSA E., FREUND J.A., HUBER M.T., VOIGT K., MOSS F., BRAUN H.A., FEUDEL U. Noisy activation kinetics induces bursting in the Huber-Braun neuron model. The European Physical Journal-Special Topics. 2010, 187(1), pp. 199-203, doi: 10.1140/epjst/e2010-01284-1.

[9] FINKE C., FREUND J.A., ROSA E., BRYANT P.H., BRAUN H.A., FEUDEL U. Temperature-dependent stochastic dynamics of the Huber-Braun neuron model. Chaos: An Interdisciplinary Journal of Nonlinear Science. 2011, 21(4), 047510, doi: 10.1063/1.3668044.

[10] HAN C., WANG J., DENG B. Fire patterns of modified HH neuron under external sinusoidal ELF stimulus. Chaos, Solitons 8 Fractals. 2009, 41(4), pp. 2045-2054, doi: 10.1016/j. chaos. 2008.08.011.

[11] HARISH O., GOLOMB D. Control of the firing patterns of vibrissa motoneurons by modulatory and phasic synaptic inputs: a modeling study. Journal of neurophysiology. 2010, 103(5), pp. 2684-2699, doi: 10.1152/jn.01016. 2009.

[12] HENRY C. Spike trains in a stochastic Hodgkin-Huxley system. BioSystems. 2005, 80(1), pp. 25-36, doi: 10.1016/j. biosystems. 2004.09.032.

[13] HORIKAWA Y. A spike train with a step change in the interspike intervals in the FitzHughNagumo model. Physica D: Nonlinear Phenomena. 1995, 82(4), pp. 365-370, doi: 10.1016/ 0167-2789 (95) 00049-A.

[14] HUBER M.T., BRAUN H.A. Conductance versus current noise in a neuronal model for noisy subthreshold oscillations and related spike generation. Biosystems. 2007, 89(1), pp. 38-43, doi: $10.1016 / \mathrm{j}$. biosystems. 2006.05.009.

[15] IZHIKEVICH E.M. Resonate-and-fire neurons. Neural networks. 2001, 14(6), pp. 883-894, doi: $10.1016 / \mathrm{S} 0893-6080$ (01) 00078-8.

[16] JIN W.-Y., XU J.-X., WU Y., HONG L. Rate of afferent stimulus dependent synchronization and coding in coupled neurons system. Chaos, Solitons 83 Fractals. 2004, 21(5), pp. 12211229, doi: $10.1016 / \mathrm{j}$. chaos. 2003.12.074.

[17] POSTNOVA S., VOIGT K., BRAUN H.A. Neural synchronization at tonic-to-bursting transitions. Journal of biological physics. 2007, 33(2), pp. 129-143, doi: 10.1007/ s10867-007-9048-x. 
Wang J. et al.: Dynamical properties of firing patterns...

[18] SHORTEN P.R., WALL D.J.N. A Hodgkin-Huxley model exhibiting bursting oscillations. Bulletin of mathematical biology. 2000, 62(4), pp. 695-715, doi: 10.1006/bulm.2000.0172.

[19] WANG J., SI W., CHE Y., FEI X. Spike trains in Hodgkin-Huxley model and ISIs of acupuncture manipulations. Chaos, Solitons 6 Fractals. 2008, 36(4), pp. 890-900, doi: 10. $1016 / j$.chaos . 2006.07.010.

[20] WANG L., LIU S., ZHANG L., ZENG Y. Dynamical properties of firing patterns in ELI pyramidal neuron under external electric field stimulus. Neurological Sciences. 2013, 34(9), pp. 1517-1522, doi: 10.1007/s10072-012-1270-z.

[21] XUE J.X., YUNFAN G., WEI R., SANJUE H., FUZHOU W. Propagation of periodic and chaotic action potential trains along nerve fibers. Physica D: Nonlinear Phenomena. 1997, 100(1), pp. 212-224, doi: 10.1016/S0167-2789(96)00188-1. 